Article

\title{
Preparation and Photocatalytic Properties of CdS and ZnS Nanomaterials Derived from Metal Xanthate
}

\author{
Neli Mintcheva ${ }^{1,2, *(\mathbb{D})}$, Gospodinka Gicheva ${ }^{2}$, Marinela Panayotova ${ }^{2}$, Wilfried Wunderlich ${ }^{3}(\mathbb{D}$, \\ Aleksandr A. Kuchmizhak ${ }^{4,5}$ and Sergei A. Kulinich 1,6,*(D) \\ 1 Research Institute of Science and Technology, Tokai University, Hiratsuka, Kanagawa 259-1292, Japan \\ 2 Department of Chemistry, University of Mining and Geology, Sofia 1700, Bulgaria; \\ e_gospodinka@yahoo.com (G.G.); marichim@mgu.bg (M.P.) \\ 3 Department of Materials Science, Tokai University, Hiratsuka, Kanagawa 259-1292, Japan; \\ wi-wunder@rocketmail.com \\ 4 Far Eastern Federal University, 690041 Vladivostok, Russia; alex.iacp.dvo@mail.ru \\ 5 Institute of Automation and Control Processes, Far Eastern Branch of the Russian Academy of Science, \\ 690091 Vladivostok, Russia \\ 6 Department of Mechanical Engineering, Tokai University, Hiratsuka, Kanagawa 259-1292, Japan \\ * Correspondence: nnmintcheva@mgu.bg (N.M.); skulinich@tokai-u.jp (S.A.K.)
}

Received: 17 September 2019; Accepted: 9 October 2019; Published: 11 October 2019

\begin{abstract}
In this paper, we report a new, simple method for the synthesis of CdS and ZnS nanoparticles (NPs) prepared in a basic aqueous medium using metal xanthate as the sulfur source. The structure, morphology, size distribution, optical band gap, and photocatalytic properties of the newly obtained nanomaterials were investigated by UV-Vis spectroscopy, X-ray diffraction, and transmission electron microscopy. The results show that both CdS and ZnS crystallized in cubic phase and formed NPs with average sizes of 7.0 and $4.2 \mathrm{~nm}$ for CdS and ZnS, respectively. A blue shift of UV-Vis absorbance band and higher energy band gap values were observed for both materials in comparison with their bulk counterparts, which is in accordance with the quantum confinement effect. The as-prepared nanomaterials were tested in visible-light driven photocatalytic decomposition of methylene blue (MB). After irradiation for $180 \mathrm{~min}$, the degradation rate of MB with a concentration of $8 \times 10^{-6} \mathrm{~mol} / \mathrm{L}$ mixed with a photocatalyst (CdS or ZnS, both $10 \mathrm{mg}$ in $100 \mathrm{~mL}$ solution of MB) was found to be $72 \%$ and $61 \%$, respectively. The CdS NPs showed better photocatalytic activity than ZnS, which could be explained by their lower energy band gap and thus the ability to absorb light more efficiently when activated by visible-light irradiation.
\end{abstract}

Keywords: ZnS nanoparticles; CdS nanoparticles; xanthate precursor; photocatalysis; visible-light activation

\section{Introduction}

Semiconductor nanoparticles (NPs) demonstrate unique optical, electronic, photoluminescence, and photocatalytic properties that differ from those of their bulk counterparts because many of these properties are size-dependent at the nanoscale. Such a dependence arises from the change in the surface-to-volume ratio, and as a result of the quantum confinement effect at small sizes. The latter effect consists of the widening of the HOMO-LUMO gap with size reduction, which causes an increase in energy band gap and blue shift of the excitonic absorption peak of NPs [1,2]. The resulting changes in optical and photocatalytic properties were widely investigated for many semiconductors, including compounds of the II-VI class, i.e., those with $\mathrm{Zn}^{2+}, \mathrm{Cd}^{2+}$, and $\mathrm{Hg}^{2+}$ as cations and $\mathrm{O}^{2-}, \mathrm{S}^{2-}$, $\mathrm{Se}^{2-}$, and $\mathrm{Te}^{2-}$ as anions. As typical examples, the photocatalytic activities of $\mathrm{ZnO}, \mathrm{ZnS}$, and CdS 
nanomaterials draw a lot of attention owing to their environmental and energy-related applications, such as degradation of organic pollutants, hydrogen production, catalytic $\mathrm{CO}_{2}$ conversion, etc. [3-6].

Nanoparticles of metal sulfides, such as CdS and ZnS, are believed to be effective photocatalysts due to their proper band gaps, the rapid formation of electron-hole pairs, and the enhanced adsorption of target molecules on the surface $[5,6]$. The photocatalytic performance of metal sulfides can be improved through more efficient light absorption and the reduction of hole-electron recombination-both properties being dependent on the band gap energy and crystal structure of the material. In turn, the band gap of the nanomaterial is known to be influenced not only by its particle size, but also by its composition, morphology, and structural defects. Therefore, the photocatalytic properties of semiconductor NPs can be altered via different synthetic routes, aiming at products with well-controlled size, shape, and crystallinity. That is why various approaches have been developed so far to provide metal sulfides with specific characteristics and properties $[5,6]$, including controlled precipitation in the presence of thiourea $[7,8]$ or sodium sulfide [9], hydrothermal reduction of sulfur [10], etc.

There are also chemical methods that rely on the thermal decomposition of molecular precursors that give rise to metal sulfide NPs. Many metal complexes with S-containing molecules as ligands, such as dithiocarbamate [11-13], thiosemicarbazone [14,15], thiobenzoates [16], glutathione [17], and thiourea [18], were applied for the preparation of various metal sulfide NPs with tunable morphology and optical properties. Metal xanthates (dithiocarbonates) are also used as single precursors for solvothermal processes [19,20], the hot-injection method [21], thermolysis in dimethylfolmamide [22,23], etc. Singhal et al. reported the formation of palladium sulfide from the thermal decomposition of square-planar $\operatorname{Pd}(\mathrm{II})$ xanthate complexes in dioctyl ether [24]. Efrima et al. used long-chain alkyl xanthates (hexadecyl xanthate) as a precursor for metal sulfides and achieved a controllable NP size at a variety of temperatures and xanthate concentrations [25]. The same group found that electron-donating solvents, such as alkylamines, permitted the lowering of the reaction temperature and formed NPs under mild conditions [26]. Similarly, other authors used ethylene diamine as a capping and coordinating agent to favor the formation of sulfide NPs [19,20].

The existing synthetic methods are often reported to require high medium temperatures (and thus special equipment) [20,21], to use toxic organic solvents and/or surfactants [22-26], and to take a relatively long reaction time [19]. Therefore, in this work, we propose a new method for preparing $\mathrm{CdS}$ and ZnS nanomaterials that has significant advantages over previously developed approaches: (1) low reaction temperature and easy handling of the process, (2) surfactant-free route that produces minimum waste chemicals, (3) good yield and production rate of both CdS and ZnS NPs with distinctive morphology and photocatalytic performance.

In this paper, we report the easy synthesis of metal sulfide (CdS and ZnS) NPs from xanthate as a sulfur source without the use of any organic solvents or surfactants. The reaction is carried out under mild conditions $\left(60-80^{\circ} \mathrm{C}\right)$ and in a basic water medium where metal xanthates decompose to sulfide ions and the formed metal sulfides crystallize into a low-temperature cubic phase. Both produced materials are shown to be nano-sized, with average particle sizes around 7.0 and $4.2 \mathrm{~nm}$ (for CdS and $\mathrm{ZnS}$, respectively), and demonstrate photocatalytic activity towards methylene blue (MB) under visible light irradiation.

\section{Materials and Methods}

As the first stage of the experiments, reaction conditions (such as temperature, time, and ratio between reagents) were optimized in order to obtain pure phases of $\mathrm{ZnS}$ and CdS without coprecipitation of other compounds. The effect of the organic group in xanthate on the process kinetics and structure of the obtained product was also investigated, with the results being reported elsewhere [27]. Ethylxanthate and amylxanthate were found to be the most suitable sulfur precursors to produce single-phase ZnS NPs, while the use of butyl- and hexylxanthates was associated with the formation of side products, and required a longer reaction time and a higher temperature for the decomposition 
of xanthate and the formation of metal sulfide. That is why, below, we mainly focus on the products prepared with the help of ethylxanthate, which served as a source of sulfur anions in alkaline media.

\subsection{Reagents Used}

Potassium ethylxanthate, $\mathrm{C}_{2} \mathrm{H}_{5} \mathrm{OCS}_{2} \mathrm{~K}$ (Acros organics, Geel, Belgium), $\mathrm{Cd}\left(\mathrm{NO}_{3}\right)_{2}$ (Teocom, Sofia, Bulgaria), $\mathrm{Zn}\left(\mathrm{NO}_{3}\right)_{2} \cdot 6 \mathrm{H}_{2} \mathrm{O}$ (Teocom, Sofia, Bulgaria), $\mathrm{KOH}$ (Teocom, Sofia, Bulgaria), and ethanol (Teocom, Sofia, Bulgaria) were analytical grade reagents and were used as supplied, without further purification.

\subsection{Preparation of $\mathrm{ZnS}$}

An aqueous solution of potassium ethylxanthate $(0.400 \mathrm{~g}$ or $0.0025 \mathrm{~mol}))$ in $40 \mathrm{~mL}$ of distilled water and $\mathrm{KOH}(0.200 \mathrm{~g}$, or $0.0036 \mathrm{~mol})$ in $5 \mathrm{~mL}$ of water were mixed and stirred at $65^{\circ} \mathrm{C}$ for $1 \mathrm{~h}$. Then a solution of zinc nitrate, $\mathrm{Zn}\left(\mathrm{NO}_{3}\right)_{2} \cdot 6 \mathrm{H}_{2} \mathrm{O}(0.7438 \mathrm{~g}$, or $0.0025 \mathrm{~mol})$, dissolved in $12 \mathrm{~mL}$ of distilled water, was slowly added to the basic solution of potassium ethylxanthate and the resulting mixture was heated at $80^{\circ} \mathrm{C}$ for $3 \mathrm{~h}$. After cooling, the precipitate was centrifuged and washed several times with water and ethanol. Finally, the obtained material was dried overnight in air and then in an oven at $230{ }^{\circ} \mathrm{C}$ for $2 \mathrm{~h}$.

\subsection{Preparation of $C d S$}

An aqueous solution of $\mathrm{Cd}\left(\mathrm{NO}_{3}\right)_{2}(0.7712 \mathrm{~g}$, or $0.0025 \mathrm{~mol})$ in $12 \mathrm{~mL}$ of distilled water was added dropwise into $45 \mathrm{~mL}$ of a warm basic solution of potassium ethylxanthate $(0.400 \mathrm{~g}, 0.0025 \mathrm{~mol})$ and $\mathrm{KOH}(0.200 \mathrm{~g}, 0.0036 \mathrm{~mol})$. All the procedures and steps were the same as those used for the preparation of ZnS NPs.

\subsection{Characterization of the Products}

The as-prepared materials were redispersed in ethanol and their UV-Vis spectra were measured. A drop of each colloid was placed onto a $\mathrm{Cu}$ grid and examined by transmission electron microscopy (TEM). The X-ray diffraction (XRD) patterns of the powder samples were recorded to evaluate their phase composition. The following instruments were used to characterize the synthesized nanomaterials: UV-Vis spectrophotometer (BOECO S-220, Hamburg, Germany), X-ray diffractometer (BRUKER D2 Phaser, $\mathrm{Cu} / \mathrm{Ni}$ radiation, $\lambda=0.154184 \mathrm{~nm}$, Karlsruhe, Germany), and TEM microscope operated at $200 \mathrm{kV}$ (Hitachi, HF $2200 \mathrm{TU}$, Tokyo, Japan).

\subsection{Photocatalytic Properties}

The photocatalytic activity of both nanomaterials, CdS and ZnS, was evaluated via photodegradation of MB under visible-light irradiation. The tests were carried out by using $100 \mathrm{~mL}$ of aqueous $\mathrm{MB}$ with concentration $8 \times 10^{-6} \mathrm{~mol} / \mathrm{L}$ and $10 \mathrm{mg}$ of photocatalyst, the dispersion being stirred and irradiated with a $1000 \mathrm{~W}$ tungsten-halogen lamp for $180 \mathrm{~min}$. Prior to irradiation, the reaction mixture was kept in the dark for $30 \mathrm{~min}$ to reach the adsorption-desorption equilibrium between the dye molecules and the catalyst surface. The decomposition of MB was monitored by following the absorption changes of its characteristic band at $664 \mathrm{~nm}$ in UV-Vis spectra [28]. Every $30 \mathrm{~min}$, an aliquot of $4 \mathrm{~mL}$ was pipetted, centrifuged to precipitate the NPs, and the UV-Vis spectrum of the liquid was recorded, after which the aliquot was sonicated and returned back to the main volume.

\subsection{Equations Used}

Based on the UV-Vis absorption spectra, energy band gaps were estimated using the following Tauc relation:

$$
\alpha h v=A\left(h v-E_{\mathrm{g}}\right)^{n},
$$


where $\alpha$ is the absorption coefficient, $h v$ is the photon energy, $E_{\mathrm{g}}$ is the band gap, $n=1 / 2$ for direct band gap transition, and $A$ is a constant that is different for different transitions [18,29].

\section{Results and Discussion}

\subsection{Formation of $\mathrm{ZnS}$ and $\mathrm{CdS}$}

Metal xanthates with the general formula ROCSSM (where $\mathrm{M}$ is a metal cation and $\mathrm{R}=\mathrm{C}_{2} \mathrm{H}_{5}$-, $\mathrm{C}_{3} \mathrm{H}_{7-}, \mathrm{C}_{4} \mathrm{H}_{9-}, \mathrm{C}_{5} \mathrm{H}_{11^{-}}$, or $\left.\mathrm{C}_{6} \mathrm{H}_{13^{-}}\right)$are salts of their corresponding xanthic acids. Their solubility depends on the length of the carbon chain in $\mathrm{R}$ and the nature of the metal ion M. Sodium and potassium salts are soluble in water, while transition metals form slightly soluble products.

The dissociation of such salts in water leads to the formation of a xanthate anion that has two resonance structures:<smiles>[R]OC(=S)[As]C#[SH]</smiles>

The xanthate ion is unstable in both acidic and basic solutions. In an alkaline medium, it undergoes hydrolysis to carbonate, alcohol, carbon disulfide, and trithiocarbonate (Equation (1)). The $\mathrm{CS}_{3}{ }^{2-}$ anion hydrolyzes further to carbonate and hydrogen sulfide (Equation (2)), whereas carbon disulfide forms carbon dioxide and hydrogen sulfide (Equation (3)). The processes are accompanied by the deprotonation of hydrogen sulfide to sulfide ion in an alkaline medium (Equation (4)) [30,31]. The release of $\mathrm{S}^{2-}$ ions allows for the controllable formation of metal sulfide NPs (Equation (5)) under particular experimental conditions that were optimized and established in our preliminary study. Here, we treated potassium xanthate in an excess of potassium hydroxide (ratio 1:1.4) at $65^{\circ} \mathrm{C}$, after which $\mathrm{Zn}^{2+}\left(\right.$ or $\mathrm{Cd}^{2+}$ ) ions were added to prepare $\mathrm{ZnS}$ (or CdS) NPs.

$$
\begin{gathered}
6 \mathrm{ROCS}_{2}^{-}+3 \mathrm{H}_{2} \mathrm{O} \leftrightarrow \mathrm{CO}_{3}^{2-}+6 \mathrm{ROH}+3 \mathrm{CS}_{2}+2 \mathrm{CS}_{3}^{2-} \\
\mathrm{CS}_{3}^{2-}+3 \mathrm{H}_{2} \mathrm{O} \leftrightarrow \mathrm{CO}_{3}^{2-}+3 \mathrm{H}_{2} \mathrm{~S} \\
\mathrm{CS}_{2}+2 \mathrm{H}_{2} \mathrm{O} \leftrightarrow \mathrm{CO}_{2}+2 \mathrm{H}_{2} \mathrm{~S} \\
\mathrm{H}_{2} \mathrm{~S}+2 \mathrm{OH}^{-} \leftrightarrow \mathrm{S}^{2-}+2 \mathrm{H}_{2} \mathrm{O} \\
S_{(a q)}^{2-}+\mathrm{M}_{(a q)}^{2+} \leftrightarrow \mathrm{MS}_{(s)}, \quad M=\mathrm{Cd}^{2+}, \mathrm{Zn}^{2+}
\end{gathered}
$$

\subsection{Characterization of Nanomaterials}

The composition and crystal structure of newly obtained ZnS and CdS nanomaterials were studied by means of powder XRD, with the obtained patterns being shown in Figure 1. The cubic form (zinc blend type) of CdS shows characteristic Bragg reflections at $2 \theta$ values of $26.5^{\circ}, 30.6^{\circ}, 43.9^{\circ}$, and $52.0^{\circ}$, corresponding to the planes with Miller indices (111), (200), (220), and (311), respectively, as indicated in the reference pattern simulated from the database (ICSD 252373) [32]. The peaks observed for the as-prepared CdS NPs (see the red pattern in Figure 1) match the reference data well, which is why the CdS NPs were assigned to a cubic phase that is metastable at room temperature. The same crystal phase was reported by other authors who synthesized CdS in a basic medium from cadmium chloride using thiourea as a $\mathrm{S}^{2-}$ source $[7,18]$. The peak positions at $29.0^{\circ}, 48.6^{\circ}$, and $57.4^{\circ}$ observed in the pattern of the as-prepared ZnS NPs (the black line in Figure 1) are assigned to the reflections of the (111), (220), and (311) planes of the cubic ZnS structure, consistent with previously published data for the ZnS crystal with a cell parameter of $5.38 \AA$ and a cell volume of $155.97 \AA^{3}$ (space group F-4 3m) (ICSD 52223) [33]. The broadening of observed diffraction peaks in both patterns in Figure 1 is due to the small sizes of analyzed NPs $[7,8]$. 


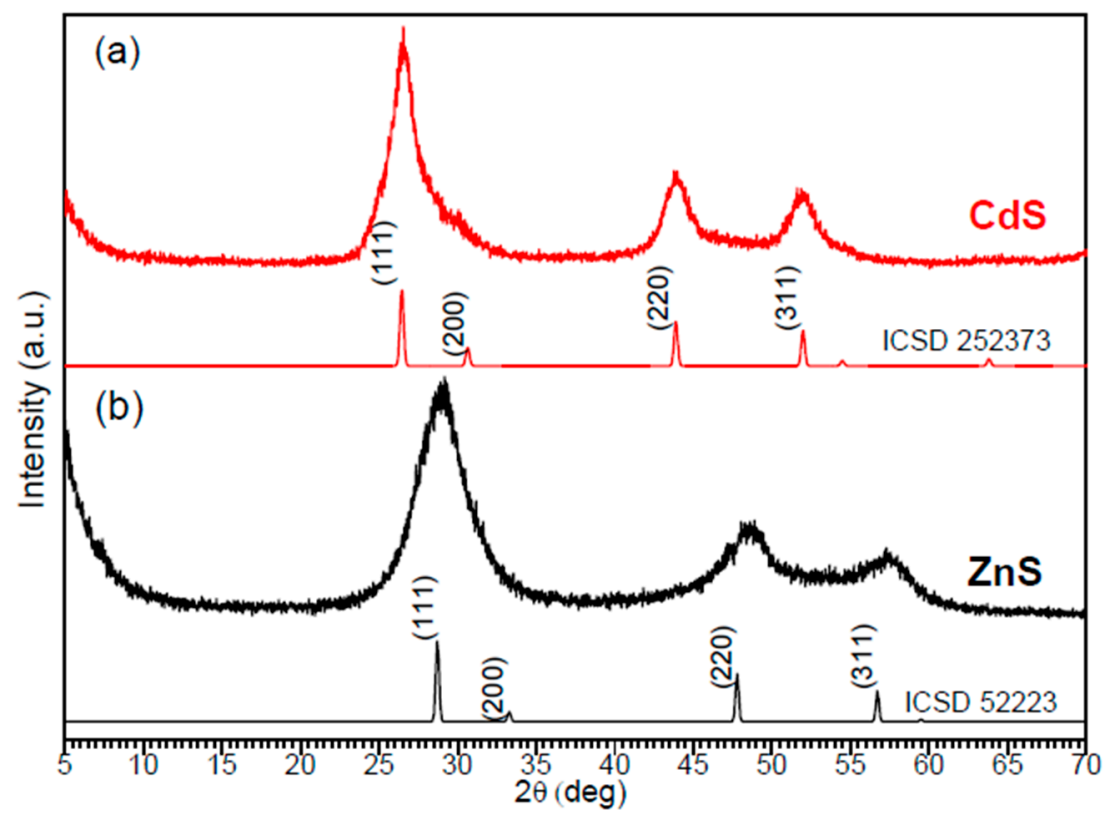

Figure 1. XRD diffraction patterns of (a) as-prepared CdS and simulated XRD pattern of cubic CdS (ICSD 252373); and (b) as-prepared ZnS and simulated XRD pattern of cubic ZnS (ICSD 52223).

The morphology of the as-prepared samples was characterized by SEM and TEM, with the micrographs of CdS and ZnS NPs being displayed in Figures 2 and 3, respectively. Spherical NPs with quite a narrow size distribution can be seen in the SEM and TEM images for both metal sulfides. The analysis of high-resolution images permitted us to determine the NP size, which is in the range of 5.0-9.0 nm (CdS, Figure 3c) and 2.6-5.6 nm (ZnS, Figure 3d). The average diameter of CdS NPs $(7.0 \mathrm{~nm})$ was thus found to be somewhat bigger than that of ZnS NPs $(4.2 \mathrm{~nm})$, which may be attributed to the lower solubility product for CdS $\left(\mathrm{K}_{\mathrm{sp}}(\mathrm{CdS})=1.0 \times 10^{-28}\right)$ in comparison with the value for $\mathrm{ZnS}$ $\left(\mathrm{K}_{\mathrm{sp}}(\mathrm{ZnS})=1.0 \times 10^{-23}\right)$ [34]. This can explain why, when the concentrations of $\mathrm{Cd}^{2+}\left(\mathrm{Zn}^{2+}\right)$ and $\mathrm{S}^{2-}$ ions in solution are almost the same, the heterogeneous equilibrium presented in Equation (5) shifts towards the solid phase of the metal sulfide and causes faster crystal growth, leading to the formation of larger NPs of CdS than of ZnS.
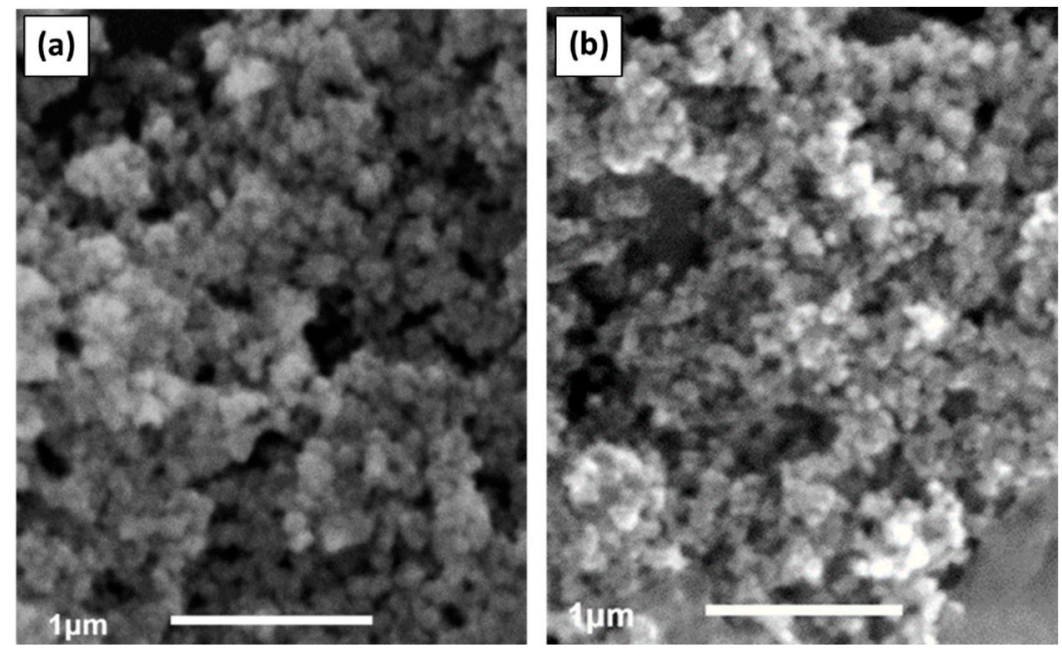

Figure 2. SEM images of as-prepared samples (a) CdS and (b) ZnS. 

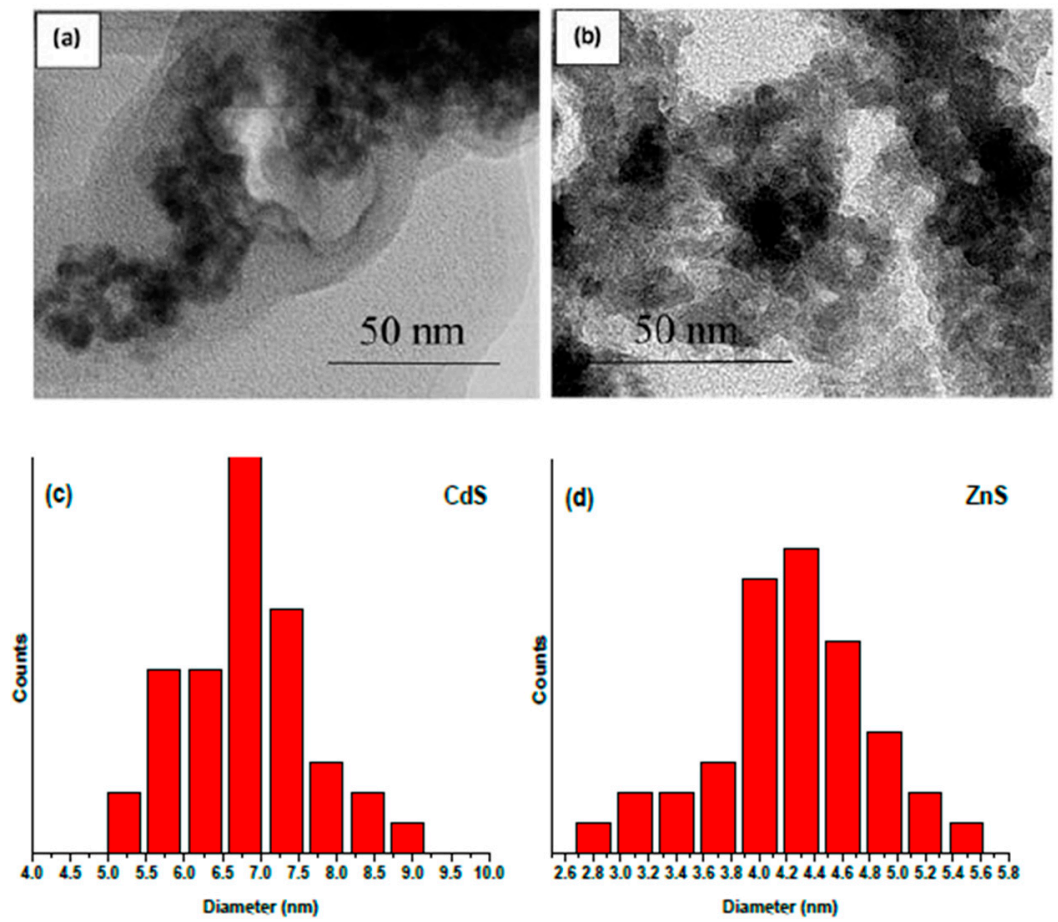

Figure 3. TEM images of as-prepared samples: (a) CdS and (b) ZnS. Histograms with size distribution for (c) CdS and (d) ZnS NPs.

The UV-Vis spectra of CdS and ZnS NPs dispersed in ethanol are displayed in Figure 4a,b. The spectrum of CdS colloid shows a wide quantum excitonic shoulder at around $470 \mathrm{~nm}$, which is followed by a rise of absorption and a strong peak below $300 \mathrm{~nm}$ in the UV region (see panel (a)). The absorption threshold for bulk CdS is known to be around $490 \mathrm{~nm}$ [35]. The profile of the UV-Vis spectrum of ZnS colloid is very similar to that of CdS (compare panels (a) and (b) in Figure 4), which is not surprising given the structural and electronic similarity of both sulfides. In the case of $\mathrm{ZnS}$, the absorption peak moves to $310 \mathrm{~nm}$, while for bulk $\mathrm{ZnS}$ it equals $336 \mathrm{~nm}$ [36]. It is clear that the observed blue shift of the absorption band for both ZnS and CdS NPs is explained by the quantum size effect, which is realized when NPs with sizes comparable to or smaller than the Bohr exciton radius are formed [1,36]. Accordingly, the band gap energies of both CdS and ZnS NPs were found to increase in comparison with their bulk materials. The band gap values were evaluated from the Tauc plot of $(\alpha h v)^{2}$ against photon energy, $h v$, as is shown in Figure 4 (insets). The $E_{\mathrm{g}}$ values were determined at the interception of the $X$-axis and the extrapolated tangent to the linear part of the curves, following a standard protocol reported elsewhere [29]. The band gaps were estimated to be 3.1 and $4.0 \mathrm{eV}$ for CdS and ZnS NPs, respectively, while their corresponding values for bulk materials are equal to $2.53 \mathrm{eV}$ (CdS) and $3.68 \mathrm{eV}(\mathrm{ZnS})[36,37]$.
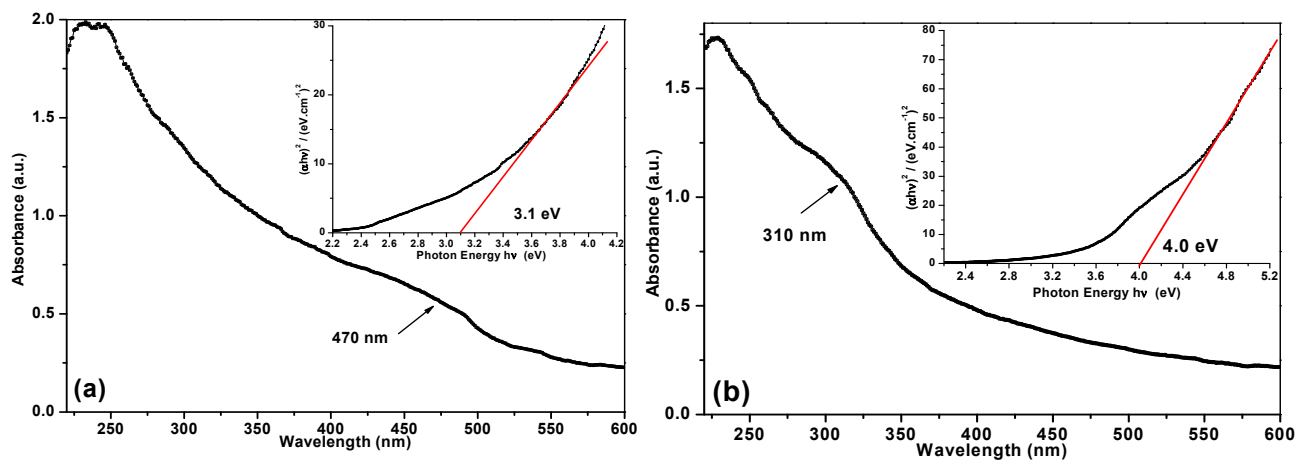

Figure 4. UV-Vis spectra and Tauc plots (as insets) of (a) CdS and (b) ZnS colloids in ethanol. 


\subsection{Photocatalytic Tests}

Photodegradation of MB under visible-light irradiation in the presence of CdS (or ZnS) NPs (as a suspension) was carried out for a period of $3 \mathrm{~h}$. In order to reach adsorption-desorption equilibrium of MB on the NP surface, a dark phase (indicated in Figure 5 with a dashed line) was first applied for $30 \mathrm{~min}$, during which a slight decrease in $\mathrm{MB}$ concentration was observed due to the adsorption of the dye on the catalyst's surface. The changes in MB concentration caused by its photocatalytic degradation were tracked by means of UV-Vis spectroscopy. It was found that the absorbance of MB at $664 \mathrm{~nm}$ (where its strongest peak is located) decreased over time in the presence of both newly prepared nanomaterials. Figure 5 compares the performance of both new nanomaterials (black and red lines) with a blank MB sample (blue line) that was traced under the same conditions, but without any photocatalyst being added. As seen in Figure 5, after $3 \mathrm{~h}$, the dispersion with CdS NPs lost $~ 72 \%$ of its $\mathrm{MB}$ and the ZnS NPs lost $\sim 61 \%$ of MB, whereas in the blank sample less than $13 \%$ of MB decolorized.

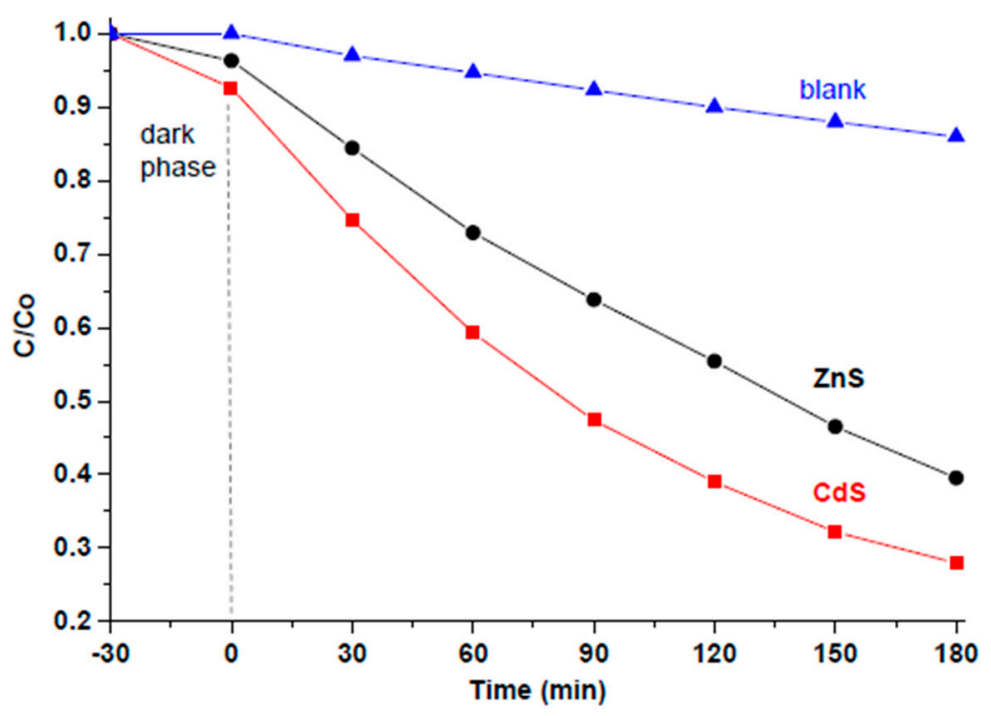

Figure 5. Photocatalytic activity of $\mathrm{CdS}$ and $\mathrm{ZnS}$ towards $\mathrm{MB}$ degradation in aqueous solution. Decay of MB caused by ZnS (red) and CdS (black line) is clearly observed over time.

It should be noted that, normally, a direct comparison between two different nanomaterials (with different particle sizes and chemical composition) is difficult, at least because their active surface exposed to the dye and the number of active surface sites are different. In this study, for simplicity we used equal masses of catalysts $(10 \mathrm{mg}$ of nanomaterial in $100 \mathrm{~mL}$ of aqueous $\mathrm{MB}$ with concentration $8 \times 10^{-6} \mathrm{~mol} / \mathrm{dm}^{3}$, which corresponds to $8 \times 10^{-7} \mathrm{~mol}$ of MB per $10 \mathrm{mg}$ of catalyst). Therefore, considering that the molar mass of CdS is larger than that of $\mathrm{ZnS}$, the amount of CdS material (in mol) was smaller. Nevertheless, it is clearly seen in Figure 5 that CdS NPs decayed the MB molecules faster and more efficiently than their $\mathrm{ZnS}$ counterparts. Therefore, it is obvious that, of the two sulfide nanomaterials prepared in this work, the sample based on CdS demonstrated higher photocatalytic activity.

The degradation of $\mathrm{MB}$ catalyzed by semiconductor materials is known to be a first-order reaction with respect to $\mathrm{MB}$, and the rate is proportional to the concentration of $\mathrm{MB}$ (rate $=k[M B])[38,39]$. Therefore, the rate constant, $k$, can be determined as a slope of $\ln \left(C / C_{0}\right)$ plotted against time, as seen in Figure 6. For room temperature $(300 \mathrm{~K})$, the estimated rate constants of CdS- and ZnS-catalyzed reactions were found to be $k_{\mathrm{CdS}}=6.8 \times 10^{-3} \mathrm{~min}^{-1}$ and $k_{\mathrm{ZnS}}=4.9 \times 10^{-3} \mathrm{~min}^{-1}$, respectively. Both values are in good agreement with those previously reported for similar photocatalysts by others [40]. It is worth noting that our nanomaterials demonstrated 2-4-fold higher rate constants and better degradation efficiency than CdS and ZnS obtained by other methods and tested under similar conditions [40]. The high photocatalytic performance of both metal sulfides prepared through 
the xanthate-mediated method can be explained by the formation of NPs enriched with sulfur vacancies $[9,41]$. Such vacancies are believed to promote visible light absorption, which is observed as a tail above $400 \mathrm{~nm}(\mathrm{ZnS})$ and $500 \mathrm{~nm}(\mathrm{CdS})$ in the absorbance spectra of both materials (Figure 4a,b). They can also entrap photogenerated electrons and restrain the electron-hole recombination, thus increasing the photocatalytic activity of such nanomaterials. As mentioned above, the band gap for $\mathrm{CdS}$ is $3.1 \mathrm{eV}$, which is lower than that of $\mathrm{ZnS}(4.0 \mathrm{eV})$. Therefore, CdS NPs need lower energy to excite their electrons from valence to conductive band and to generate exciton electron-hole pairs. As a result, such NPs could absorb the visible light used for irradiation more efficiently, which in turn led to their higher photocatalytic activity.

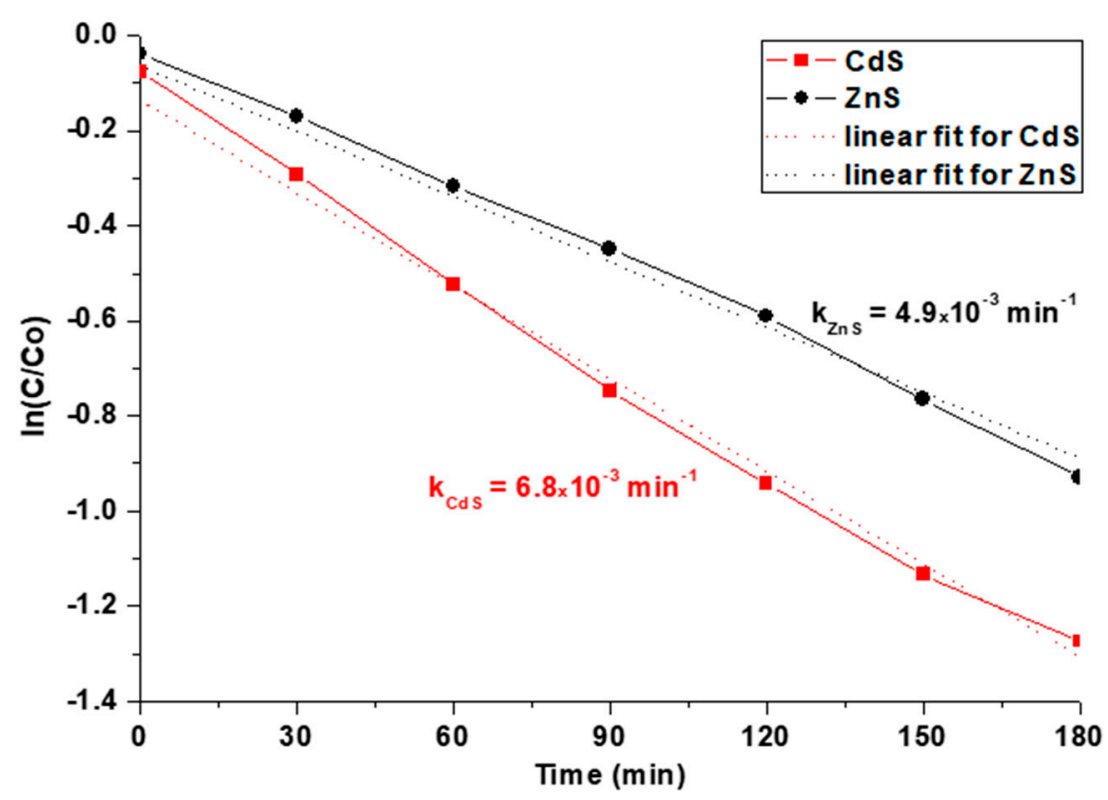

Figure 6. Linear fit of $\ln \left(C / C_{0}\right)$ as a function of time for the first-order reaction with respect to $M B$.

\section{Conclusions}

This work demonstrates, for the first time, the preparation of CdS and ZnS nanomaterials via a slow and controllable decomposition of xanthate in alkaline aqueous solution. Such reaction conditions are shown to ensure the formation of nanoparticles with a cubic crystal structure, small particle sizes, narrow size distribution, and sulfur vacancies for both metal sulfides. The newly prepared nanomaterials revealed high photocatalytic performance, efficiently decaying methylene blue in an aqueous solution under visible-light irradiation. This property results from the unique structure of the novel xanthate-mediated nanomaterials, with numerous structural defects that reduce the hole-electron recombination rate and thus assist in the formation of oxidizing species that react with organic molecules. Even though the CdS nanoparticles had larger sizes $(7.0 \mathrm{~nm}$ on average), they outperformed their ZnS counterparts (with average size $4.2 \mathrm{~nm}$ ) as photocatalyst. This is explained by the smaller band gap found for the CdS nanomaterial, which could absorb visible light more efficiently.

Author Contributions: N.M. and G.G. designed and carried out the experiments; N.M., M.P. and W.W. conducted most of the analyses. N.M., A.A.K. and S.A.K. wrote the manuscript. All the authors discussed the results and helped prepare the manuscript.

Funding: This work was supported by an exchange academic grant from the Tokai University (Japan) and the National Science Fund of Bulgaria (grant no. DN-17/20). A.K. expresses his gratitude to the Ministry of Science and Higher Education of the Russian Federation (grant no. MK-3258.2019.8).

Conflicts of Interest: The authors declare no conflict of interest. 


\section{References}

1. Bera, D.; Qian, L.; Tseng, T.-K.; Holloway, P.H. Quantum dots and their multimodal applications: A review. Materials 2010, 3, 2260-2345. [CrossRef]

2. Edvinsson, T. Optical quantum confinement and photocatalytic properties in two-, one- and zero-dimensional nanostructures. R. Soc. Open Sci. 2018, 5, 180387. [CrossRef] [PubMed]

3. Xu, C.; Anusuyadevi, P.R.; Aymonier, C.; Luque, R.; Marre, S. Nanostructures materials for photocatalysis. Chem. Soc. Rev. 2019, 48, 3868-3902. [CrossRef] [PubMed]

4. Lee, S.L.; Chang, C.J. Recent Progress on metal sulfide composite nanomaterials for photocatalytic hydrogen production. Catalysts 2019, 9, 457. [CrossRef]

5. Lee, G.-J.; Wu, J.J. Recent developments in ZnS photocatalysts from synthesis to photocatalytic applications-A review. Powder Technol. 2017, 318, 8-22. [CrossRef]

6. Cheng, L.; Xiang, Q.; Liao, Y.; Zhang, H. CdS-Based photocatalysts. Energy Environ. Sci. 2018, 11, $1362-1391$. [CrossRef]

7. Lee, H.L.; Issam, A.M.; Belmahi, M.; Assouar, M.B.; Rinnert, H.; Alnot, M. Synthesis and characterizations of bare CdS nanocrystals using chemical precipitation method for photoluminescence application. J. Nanomater. 2009, 2009, 914501. [CrossRef]

8. Nirmala Jothi, N.S.; Joshi, A.G.; Jerald Vijay, R.; Muthuvinayagam, A.; Sagayaraj, P. Investigation on one-pot hydrothermal synthesis, structural and optical properties of $\mathrm{ZnS}$ quantum dots. Mater. Chem. Phys. 2013, 138, 186-191. [CrossRef]

9. Wageh, S.; Ling, Z.S.; Xu-Rong, X. Growth and optical properties of colloidal ZnS nanoparticles. J. Cryst. Growth 2003, 255, 332-337. [CrossRef]

10. Tezuka, K.; Takagi, H.; Shan, Y.J.; Imoto, H. Simple synthesis of zinc sulfide and cadmium sulfide under hydrothermal conditions. J. Ceram. Soc. Jpn. 2011, 119, 55-59. [CrossRef]

11. Andrew, F.P.; Ajibade, P.A. Metal complexes of alkyl-aryl dithiocarbamates: Structural studies, anticancer potentials and applications as precursors for semiconductor nanocrystals. J. Mol. Struct. 2018, 1155, 843-855. [CrossRef]

12. Onwudiwe, D.C.; Ajibade, P.A. Zn(II), Cd(II) and Hg(II) complexes of N-methyl-N-phenyl dithiocarbamate as single-source precursors for the synthesis of metal sulfide nanoparticles. Mater. Lett. 2011, 65, 3258-3261. [CrossRef]

13. Onwudiwe, D.C.; Strydom, C.; Oluwafemi, O.S.; Songca, S.P. Effect of temperature on the optical and structural properties of hexadecylamine capped ZnS nanoparticles using Zinc(II) N-ethyl-N-phenyldithiocarbamate as single source precursor. Mater. Res. Bull. 2012, 47, 4445-4451. [CrossRef]

14. Pawar, A.S.; Mlowe, S.; Garje, S.S.; Akerman, M.P.; Revaprasadu, N. Zinc thiosemicarbazone complexes: Single source precursors for alkylamine capped $\mathrm{ZnS}$ nanoparticles. Inorg. Chim. Acta 2017, 463, 7-13. [CrossRef]

15. Pawar, A.S.; Masikane, S.C.; Mlowe, S.; Garje, S.S.; Revaprasadu, N. Preparation of CdS nanoparticles from thiosemicarbazone complexes: Morphological influence of chlorido and iodido ligands. Eur. J. Inorg. Chem. 2016, 2016, 366-372. [CrossRef]

16. Zhang, Z.; Lim, W.P.; Wong, C.T.; Xu, H.; Yin, F.; Chin, W.S. From metal thiobenzoates to metal sulfide nanocrystals: An experimental and theoretical investigation. Nanomaterials 2012, 2, 113-133. [CrossRef] [PubMed]

17. Liu, Y.; Yin, S.; Yang, S. Glutathione as both ligand and sulfur source for the synthesis of full-color luminescent water-soluble CdS nanocrystals. Mater. Lett. 2017, 196, 260-263. [CrossRef]

18. Tong, H.; Zhu, Y.-J. Synthesis of CdS nanocrystals based on low-temperature thermolysis of one single-source organometallic precursor. Nanotechnology 2006, 17, 845-851. [CrossRef]

19. Sun, S.; Han, Q.; Wu, X.; Zhu, J.; Wang, X. The facile synthesis of PbS cubes and $\mathrm{Bi}_{2} \mathrm{~S}_{3}$ nanoflowers from molecular precursors at room temperature. Mater. Lett. 2011, 65, 3344-3347. [CrossRef]

20. Han, Q.; Zhao, J.; Wu, L.; Zhu, J.; Wang, X. Synthesis of CdS multipods from cadmium xanthate in ethylenediamine solution. Particuology 2015, 19, 45-52. [CrossRef]

21. Nair, P.S.; Radhakrishnan, T.; Revaprasadu, N.; Kolawole, G.; O'Brien, P. Cadmium ethylxanthate: A novel single-source precursor for the preparation of CdS nanoparticles. J. Mater. Chem. 2002, 12, $2722-2725$. [CrossRef] 
22. Han, Q.; Sun, Y.; Wang, X.; Chen, L.; Yang, X.; Lu, L. Controllable synthesis of $\mathrm{Bi}_{2} \mathrm{~S}_{3}$ hierarchical nanostructures: Effect of addition method on structures. J. Alloy. Compd. 2009, 481, 520-525. [CrossRef]

23. Sun, Y.; Han, Q.; Lu, J.; Yang, X.; Lu, L.; Wang, X. Preparation of uniform $\mathrm{Bi}_{2} \mathrm{~S}_{3}$ nanoribbons at a low temperature. Mater. Lett. 2008, 62, 3730-3732. [CrossRef]

24. Singhal, A.; Dutta, D.P.; Tyagi, A.K.; Mobin, S.M.; Mathur, P.; Lieberwirth, I. Palladium(II)/allylpalladium(II) complexes with xanthate ligands: Single-source precursors for the generation of palladium sulfide nanocrystals. J. Organomet. Chem. 2007, 692, 5285-5294. [CrossRef]

25. Pradhan, N.; Efrima, S. Single-Precursor, One-pot versatile synthesis under near ambient conditions of tunable, single and dual band fluorescing metal sulfide nanoparticles. J. Am. Chem. Soc. 2003, 125, 2050-2051. [CrossRef] [PubMed]

26. Pradhan, N.; Katz, B.; Efrima, S. Synthesis of high-quality metal sulfide nanoparticles from alkyl xanthate single precursors in alkylamine solvents. J. Phys. Chem. B 2003, 107, 13843-13854. [CrossRef]

27. Mintcheva, N.; Gicheva, G.; Panayotova, M.; Kulinich, S.A. Zinc xanthates as molecular precursors for formation of $\mathrm{ZnS}$ nanoparticles. Unpublished work.

28. Mintcheva, N.; Aljulaih, A.A.; Wunderlich, W.; Kulinich, S.A.; Iwamori, S. Laser-ablated ZnO nanoparticles and their photocatalytic activity towards organic pollutants. Materials 2018, 11, 1127. [CrossRef] [PubMed]

29. Tauc, J.; Grigorovici, R.; Vancu, A. Optical properties and electronic structure of amorphous germanium. Phys. Status Solidi 1966, 15, 627-637. [CrossRef]

30. Sodium Ethyl Xanthate; Public Report; Australian Government Publishing Service: Canberra, Australia, 1995; pp. 5-16. ISBN 0644352833.

31. Khan, G.A.; Gabrielova, L.I.; Vlasova, N.S. Flotation Reagents and Their Application; Nedra: Moscow, Russia, 1986; pp. 38-46. (In Russian)

32. Falcon, H.; Tartaj, P.; Vaquero, F.; Navarro, R.M.; Fierro, J.L.G.; Bolletta, J.P.; de Paoli, J.M.; Carbonio, R.E.; Fernandez-Diaz, M.T.; Alonso, J.A. Straightforward high-pressure synthesis and characterization of indium-based thiospinels: Photocatalytic potential for hydrogen production. Eur. J. Inorg. Chem. 2016, 10, 1558-1565. [CrossRef]

33. Jamieson, J.C.; Demarest, H.H., Jr. A note on the compression of cubic ZnS. J. Phys. Chem. Solids 1980, 41, 963-962. [CrossRef]

34. Sillen, L.G.; Martell, A.E. Stability Constants of Metal-Ion Complexes. In Lange's Handbook; The Chemical Society: London, UK, 1964; pp. 8.6-8.11. Available online: http://www.wiredchemist.com/chemistry/data/ solubility-product-constants (accessed on 23 July 2019).

35. Rossetti, R.; Hull, R.; Gibson, J.M.; Brus, L.E. Excited electronic states and optical spectra of ZnS and CdS crystallites in the 15 to 50 A size range: Evolution from molecular to bulk semiconducting properties. J. Chem. Phys. 1985, 82, 552-559. [CrossRef]

36. Kumbhojkar, N.; Nikesh, V.V.; Kshirsagar, A.; Mahamunia, S. Photophysical properties of ZnS nanoclusters. J. Appl. Phys. 2000, 88, 6260-6264. [CrossRef]

37. Gaur, R.; Jeevanandam, P. Evolution of different morphologies of CdS nanoparticles by thermal decomposition of bis (thiourea) cadmium chloride in various solvents. J. Nanopart. Res. 2015, 17, 156-168. [CrossRef]

38. Zulmajdi, S.L.N.; Ajak, S.N.F.H.; Hobley, J.; Duraman, N.; Harunsani, M.H.; Yasin, H.M.; Nur, M.; Usman, A. Kinetics of photocatalytic degradation of methylene blue in aqueous dispersions of $\mathrm{TiO}_{2}$ nanoparticles under UV-LED irradiation. Am. J. Nanomater. 2017, 5, 1-6. [CrossRef]

39. Stoyanova, M.; Christoskova, S. Catalytic degradation of methylene blue in aqueous solutions over Ni- and Co- oxide systems. Cent. Eur. J. Chem. 2011, 9, 1000-1007. [CrossRef]

40. Soltani, N.; Saion, E.; Hussein, M.Z.; Erfani, M.; Abedini, A.; Bahmanrokh, G.; Navasery, M.; Vaziri, P. Visible light-induced degradation of methylene blue in the presence of photocatalytic ZnS and CdS panoparticles. Int. J. Mol. Sci. 2012, 13, 12242-12258. [CrossRef] [PubMed]

41. Wang, G.; Huang, B.; Li, Z.; Lou, Z.; Wang, Z.; Dai, Y.; Whangbo, M.-H. Synthesis and characterization of ZnS with controlled amount of $\mathrm{S}$ vacancies for photocatalytic $\mathrm{H}_{2}$ production under visible light. Sci. Rep. 2015, 5, 8544. [CrossRef]

(C) 2019 by the authors. Licensee MDPI, Basel, Switzerland. This article is an open access article distributed under the terms and conditions of the Creative Commons Attribution (CC BY) license (http://creativecommons.org/licenses/by/4.0/). 